\title{
Literatura y sensibilidad moral: el lugar de la educación universitaria en el actual contexto colombiano $^{1}$
}

Literature and moral sensibility: The status of university education in the actual Colombian context

Literatura e sensibilidade moral: o lugar da formação universitária no atual contexto colombiano

\section{Caterine Pardo Mojica ${ }^{2}$}

Para citar este artículo: Pardo, C. (2019). Literatura y sensibilidad moral: el lugar de la educación universitaria en el actual contexto colombiano. Ciudad Paz-ando, 12(1), 47-55. doi: https://doi.org/10.14483/2422278X.13647

Fecha de recepción: 03 de julio de 2018

Fecha de aprobación: 11 de marzo de 2019

\footnotetext{
1 El presente artículo hace parte de la investigación titulada: El aporte de la literatura al desarrollo de la sensibilidad moral de estudiantes universitarios en un contexto pedagógico para la construcción de paz. La cual, a partir de la pregunta sobre cómo contribuye la literatura al desarrollo de la sensibilidad moral de estudiantes universitarios en un contexto pedagógico que propicie la construcción de paz, y partiendo de los postulados teóricos de autores como Martha Nussbaum, se desarrolló una investigación desde una perspectiva cualitativa, con un enfoque hermenéutico, en la cual participaron cuatro expertos en temas de universidad y paz, cuatro maestros, y 36 estudiantes de tres universidades de Bogotá D.C., con los cuales se realizaron entrevistas, grupos focales, conversatorios de literatura y observación participante. El análisis de la información recolectada se fundamentó en el análisis del discurso. Los resultados obtenidos permitieron comprender el lugar de la literatura en la compresión social y el reconocimiento del otro, la importancia de la sensibilidad moral en la educación universitaria y el papel de la educación universitaria en la formación de estudiantes que comprendan su lugar dentro de la construcción de paz. Se concluyó con la necesidad de formar a los estudiantes universitarios políticamente desde lugares sensibles tales como la literatura, con el fin de fortalecer no solo sus saberes disciplinares, sino su forma de relacionar dichos saberes con las realidades sociales en las cuales se encuentran inmersos.

2 Docente investigadora. Licenciada en Psicología y Pedagogía; especialista en Psicología Educativa; magíster en Investigación en Problemas Sociales Contemporáneos; magíster en Educación, Pontificia Universidad Javeriana. Correo electrónico: caterinepardo@gmail.com
} 


\section{RESUMEN}

Partiendo de la pregunta sobre el papel de la educación universitaria en el contexto colombiano actual, se presenta aquí una reflexión en torno al lugar que ocupa la literatura en el desarrollo de la sensibilidad moral de estudiantes universitarios. Se abordan tres aspectos fundamentales que sitúan la discusión: se inicia resaltando el lugar de la literatura en la comprensión social y el reconocimiento del otro, igualmente se aborda la importancia de la sensibilidad moral en la educación universitaria y, por último, se destaca el papel de la educación universitaria y la formación de estudiantes que relacionen sus saberes disciplinares con las realidades sociales en las cuales se encuentran inmersos, de forma que puedan contribuir a la construcción de paz en Colombia.

Palabras clave: construcción de paz, contexto colombiano, educación universitaria, estudiante universitario, literatura, sensibilidad moral.

\section{ABSTRACT}

Starting from the question about the role that university education plays in the actual Colombian context, we show a reflection about the place that literature occupies in the development of the moral sensitivity of university students. Three fundamental aspects are dealt with, to set the discussion: we start highlighting the place that literature occupies in the social comprehension and the recognition of the other party, at the same time the importance of moral sensitivity in university education is addressed, and lastly, the paper of university education is highlighted and student's formation which relate their disciplinary knowledge with social reality which is immersed in such a way that they can contribute to the peace in Colombia.

Keywords: peace building, Colombian context, university education, university student, literature, moral sensitivity.
Partindo da pergunta sobre o papel da formação universitária no atual contexto colombiano, é apresentada aqui uma reflexão sobre o lugar que a literatura ocupa no desenvolvimento da sensibilidade moral dos universitários. São abordados três aspectos fundamentais que situam a discussão: começa por evidenciar o lugar da literatura na compreensão social e reconhecimento do outro, aborda também a importância da sensibilidade moral no ensino universitário e, por fim, destaca o papel da formação universitária e da formação de estudantes que relacionam seu saber disciplinar às realidades sociais em que estão imersos, para que possam contribuir para a construção da paz na Colômbia.

Palavras-chave: construção da paz, contexto colombiano, formação universitária, estudante universitário, literatura, sensibilidade moral. 


\section{Introducción}

Trágicamente, el hombre está perdiendo el diálogo con los demás y el reconocimiento del mundo que lo rodea, siendo que es alli donde se dan el encuentro, la posibilidad del amor, los gestos supremos de la vida.

La resistencia, Ernesto Sábato

Un maestro acaba de recibir antes de lo previsto la notificación de que se jubilará de la universidad en la que ha trabajado por un largo tiempo. Él, un maestro de literatura que enseña a sus estudiantes su misma profesión, decide en su último día no dar su cátedra de forma habitual, inicia hablándoles del sentido de dicha labor, de cómo en un año ellos serán maestros de literatura y de que quizás lo más importante no sea la literatura misma, no sea enseñar verdades absolutas acerca de ella, sino enseñar a dudar y a pensar a sus futuros estudiantes. Les da la misión de no enseñar sencillamente literatura, sino de despertar en sus estudiantes lo que él llama el dolor de la lucidez. Con esta escena inicia la película argentina Lugares comunes, del director Adolfo Aristarain que fue estrenada en el 2002. A partir de ella es posible preguntarse por el sentido de la formación de los estudiantes dentro de la universidad, ¿a qué debe darse más importancia?, ¿qué sentido tienen los saberes que se imparten en ella? y ¿cómo la formación profesional responde a las necesidades del contexto colombiano actual?

Autores como De Sousa Santos (2004) resaltan el papel de la universidad en la sociedad, en tanto esta debe responder a las necesidades socioculturales de los contextos donde se encuentra; sin embargo, en la práctica esto no suele ocurrir así, pues la universidad ha venido centrándose en temas meramente económicos, dejando entrever cómo la educación se convierte en un bien mercantil cuya finalidad es formar profesionales productivos. Es importante reconocer que el trabajo de reformar estructuralmente el sentido de la educación universitaria es una tarea de largo aliento, por lo que propuestas investigativas que busquen fortalecer la educación humanista, que respondan a las necesidades sociales y que se encaminen en la formación de profesionales que contribuyan a la sociedad, son fundamentales, puesto que cada paso que se dé en esta dirección permitirá que la universidad recobre el sentido con el cual fue creada.

Desde esta perspectiva, la universidad es entendida como una institución de carácter social, en la cual se interpreta la realidad, sirviendo de mediadora en las relaciones entre Estado y sociedad (De Sousa, 2004). Es en la universidad donde la educación superior se materializa, posibilitando a las personas el desarrollo de sus potencialidades de una manera integral, propiciando su formación académica y profesional, tal como lo contempla la
Ley 30 de 1992; de esta forma, la universidad no solo debe propiciar en sus estudiantes una buena formación profesional, sino también debe propiciar una formación como ciudadanos que les permita entender su contexto y trabajar sobre él, de allí que su formación sea integral.

Sin embargo, dicha formación no ha sido una tarea sencilla, puesto que como lo manifiesta Nussbaum (2016a), actualmente, la universidad ha centrado sus esfuerzos, no tanto en la formación ciudadana de sus profesionales, sino en responder a indicadores internacionales de calidad, que buscan más la producción capitalista que la formación humanista, por lo que el trabajo y esfuerzo en generar espacios de formación ciudadana, donde la sensibilidad moral sea el centro, es una necesidad en la educación universitaria, más teniendo en cuenta el contexto colombiano y la necesidad de formar ciudadanos que contribuyan a la generación de paz.

Perspectivas como la de Nussbaum, permiten ver las emociones como aquellas que guardan en sí mismas un conocimiento particular, que fundamenta la moral de la persona y se reflejan en la interacción del ser humano con su contexto. Nussbaum (2008) da un lugar primordial al arte para comprender las emociones centrándose en la literatura, ya que esta permite una relación no solo cognitiva, sino afectiva frente al conocimiento de las propias emociones, así, la literatura se entiende como aquella que permite la reflexión ética sobre la vida misma; por lo tanto, para Nussbaum (2008), la literatura es entendida como un instrumento de deliberación, con el que el sujeto profundiza en su intimidad emocional y puede percibir de forma clara y distinta aquello que valora. De esta forma, la literatura profundiza en la emocionalidad del sujeto permitiéndole cuestionar y problematizar todo aquello a lo que este da significado en su vida.

Nussbaum (2014) resalta la importancia de inspirar las emociones hacia propósitos comunes, ya que si se indaga sobre los principios políticos, estos requieren para materializarse y ser estables en el tiempo, de un apoyo emocional que les permita protegerse de la jerarquización, por lo que el cultivo de sentimientos como la simpatía y el amor fortalecen los principios políticos, de allí que a la hora de hablar de la formación de ciudadanos no se reduzca el discurso a meros contenidos, sino que se vinculen las emociones y el cultivo de los sentimientos para que dicha formación trascienda.

El desarrollo de la sensibilidad moral, más allá de ser un tema de estudio, se ha convertido en el último tiempo en una necesidad, dado un escenario como el actual, donde la cotidianidad de la violencia crea distancias entre los sujetos que habitan un mismo espacio. La necesidad de acortar estas brechas hace que se busquen estrategias para que los sujetos puedan reconocerse en los otros; por ello, la necesidad de pensar en formas de desarrollar la sensibilidad moral lleva a ver los espacios ficcionales como lugares donde reside la historia del país y desde 
donde pueden empezar a construirse escenarios de paz a partir las realidades sociales, políticas y culturales que ha atravesado Colombia. La literatura se presenta como un espacio de significación del sujeto, donde este puede poner en escena su historia y reconocer la de los otros como algo que no le es ajeno.

De esta manera, la literatura permitiría al sujeto entender de forma amplia su contexto y, a su vez, situarse dentro de él desde lo ficcional, con el fin de comprender su papel en la transformación social, por lo que la literatura sería entendida más allá de lo meramente estético y cobraría importancia frente a lo social, se relacionaría con los niveles ético, político y moral del sujeto, contribuyendo a la formación de los ciudadanos y acercando los discursos sobre política, Estado y ciudadanía a un nivel en el cual la emocionalidad cobra un papel fundamental. Hablar de las emociones en el campo de la política en general y de la formación en política en particular, lleva a explorar en Aristóteles, y quienes, partiendo de él, como Martha Nussbaum, dan lugar a las virtudes morales, la felicidad, los sentimientos, y la formación del carácter humano, entre otros aspectos, permitiendo entender la formación del ciudadano más allá del mero aprendizaje de normas, reglamentos, o procedimientos justos, centrándolo en lo propiamente humano y su papel en dicha formación.

El presente artículo pretende generar una reflexión en torno al lugar que ocupa la literatura en el desarrollo de la sensibilidad moral de estudiantes universitarios, teniendo como base la pregunta sobre el lugar de la educación universitaria en el contexto colombiano actual; de esta forma, en un primer momento se sitúa el lugar de la literatura dentro de la compresión social y el reconocimiento del otro, resaltando la importancia del arte como lugar de significación y compresión del sujeto y del mundo, y tomando distancia de la idea de la literatura como un simple instrumento educativo o didáctico; igualmente, se aborda la importancia de la sensibilidad moral en la educación universitaria, resaltando el papel de la educación y la formación de estudiantes que comprendan el momento sociohistórico por el cual atraviesa el país en la actualidad; por último, se presenta una reflexión en torno a la necesidad de formar a los estudiantes universitarios políticamente desde lugares sensibles, con el fin de fortalecer no solo sus saberes disciplinares, sino la forma de relacionar dichos saberes con las realidades sociales en las cuales se encuentran inmersos.

\section{La literatura dentro de la compresión social y el reconocimiento del otro}

Theodor Adorno, en su libro Teoría estética (1984), hace referencia al arte como un hecho social y estético a la vez, ya que, por una parte, se disocia a sí mismo de la realidad empírica y con ello de la múltiple complejidad que constituye a la sociedad, además, por otra parte, pertenece a aquella realidad empírica y al complejo social de la cual hace parte. De una forma u otra el arte en relación con lo social conduce a hablar inevitablemente del lenguaje, aquel que establece la relación del sujeto con el mundo.

Si se piensa en la literatura como obra de arte es posible identificar la potencialidad de esta para reconocer al sujeto y al otro, la literatura devendría de ese sujeto y la lectura de su mundo, esta sería entonces un acto eminentemente metafísico, estaría ligada a la existencia, al moldeamiento del lenguaje y la relación que establecería el sujeto con este. La literatura estaría representada en dos planos; por un lado, la del sujeto que escribe, el cual entraría en un proceso donde conjugaría su mundo y su humanidad, mismos que estarían insertos en un momento histórico y social determinado, y la del sujeto que se acerca a ella, en la cual, este conjugaría su ser y su mundo con el del que escribe para dar significado a lo que lee y construir a partir de ello una interpretación propia.

La literatura entonces no podría ser leída como un mero conjunto de palabras estructuradas, al contrario, iría más allá de ello, se instituiría como un ejercicio que pasa por la existencia de aquel que escribe y toma el lenguaje para crear mundos o realidades paralelas a su mundo, su historicidad, su contexto y lugar; y aquel que lee, el cual interpreta los mundos que se le presentan para apropiarse de ellos y realizar sus propias interpretaciones. Lo que indicaría que la literatura permite descubrir otras formas de leer el mundo, de entenderlo y de apropiarse de él; sería una lectura que no se encuentra en la superficie del mundo como tal, sino una lectura del sujeto, de lo profundo de este en relación con el mundo y otros sujetos, una lectura en la que emerge la complejidad y contrariedad de la relación del sujeto con el mundo.

De allí que la literatura se presente como un lugar desde donde puede explorarse la relación del sujeto con el mundo y con los otros, siendo esta una relación que no solo permite la existencia del sujeto mismo, sino a su vez una consciencia del mundo. La literatura como expresión social reflejaría a su vez las relaciones del sujeto con el otro, mismas que dan significado al mundo. Frente a esto, Sábato (1976) manifiesta que en la literatura está el espíritu humano, aquel en el que se plasma el pensamiento y el arte, profundizando en la existencia del ser; de esta forma, la literatura daría una mirada profunda frente a la relación del sujeto con el otro, permitiendo entender dicha relación y, a su vez, cómo influye el mundo en ella.

\section{La sensibilidad moral en la educación universitaria}

La relación del sujeto con el otro, y el reconocimiento de ese otro como parte de sí, es un proceso que no pasa meramente por la racionalidad, no es un proceso eminentemente cognitivo, sino que es a su vez un proceso emocional y sensitivo, de allí que su comprensión requiera acercar al sujeto a lenguajes sensibles que le permitan 
comprender, desde lo sensible y desde lo racional, cómo establece una relación con el mundo y con los otros. Dicha relación, hace referencia necesariamente con la vida política del sujeto y acercarse a ella implica desplazamientos a otros lugares, no solo a los establecidos para la comprensión del sujeto, sean estos la filosofía, la psicología, la sociología o la misma pedagogía.

Así, el lenguaje literario se presenta como un espacio para comprender al sujeto y, a su vez, comprender las formas en las que establece relaciones con el otro (Nussbaum, 1995). La pregunta sobre qué aspectos determinan dicha relación se encuentra estrechamente ligada con la emoción, es la emoción la que determinará, entre otras, las formas en que el sujeto establece relaciones con otros. Hablar de las emociones, implica necesariamente abordar a Aristóteles (trad. 1998), quien define las pasiones en su Ética nicomaquea como afecciones que se acompañan del placer y la pena, pasiones tales como el temor, la cólera, el odio, la envidia, la alegría, la piedad son reconocidas por Aristóteles como aquellas que permiten que las personas cambien sus juicios y sean, en cierta medida, volubles; de esta forma, la emoción, juega un papel importante en los juicios de valor que da el sujeto sobre una cosa u otra.

Desde la perspectiva de Nussbaum (2008), la emoción guarda una estrecha relación con la creencia en tanto existen elementos de las creencias que dan sentido a la emoción. Las creencias como la emoción estarían íntimamente ligadas con la inteligencia y a su vez se entenderían como capaces de ser modificadas por la racionalidad, lo que permitiría entender que las emociones guardan una estrecha relación con la capacidad cognitiva del ser humano. La emoción entonces propiciaría una comprensión moral, es así como la reacción de la emoción estaría ligada a un cierto valor que se le asigne a la acción o hecho que ocurra frente al sujeto. De esta manera el sentimiento estaría relacionado con la moralidad y no tanto la racionalidad, por lo que se podría definir, de acuerdo con Nussbaum (2010), que los sentimientos morales son aquellos que posibilitan el desarrollo cognitivo desde la infancia, permitiendo al niño amar y amarse, al tiempo que comprender el lugar del bien y el mal en sus situaciones cotidianas. El proceso de los sentimientos morales se daría desde la infancia, pero no como un proceso meramente individual, sino como un proceso que adquiere sentido en relación con los otros que rodean al niño y que irían preformando su relación política con los otros a través del crecimiento.

Es por esto por lo que se presentan, de acuerdo con Nussbaum (2010), cuatro sentimientos morales, que con el pasar del tiempo se van resignificando y complejizando cognitivamente. El primero es la empatía, la cual se evidencia en la relación que establece el niño con su madre, a medida que el niño se forma como un ser social requiere de este sentimiento para establecer vínculos interpersonales; el siguiente sentimiento es la vergüenza que se vincula a la inestabilidad en el desarrollo de la infancia, permitiéndole al niño la construcción de relaciones jerárquicas con sus padres y creando psicológicamente unos límites para no atentar contra el otro; el tercero responde a la repugnancia, el cual puede ser definido como un carácter anormal que genera impotencia ante asuntos de la vida; por último, está el pensamiento crítico que se desarrolla en la etapa de la pubertad, en la que el sujeto tiene la capacidad de comprenderse como un ser humano y social, entendiendo la reciprocidad como un elemento fundamental para reconocer al otro. Así, podría decirse que es en el pensamiento crítico donde se evidencia el desarrollo de los tres sentimientos anteriores que se irán habituando y reconceptualizando en la adultez, permitiendo la comprensión del otro como fin y no como medio.

De esta manera, los sentimientos morales, son vistos como un proceso que se da desde la niñez, y desde donde la conciencia del otro permite los vínculos que puedan establecerse con él, es por esto que se podría decir que desde la familia se establecen ciertos sentimientos morales que de una forma $u$ otra responden a un proyecto político en medio del cual crece el niño y que puede variar de un lugar a otro. Es así como los sentimientos morales guardan relación con la vida pública y se fortalecen durante todo el ciclo de vida del ser humano, a medida que este se relaciona con otros, los sentimientos entonces adquieren sentido a través de la experiencia del sujeto con el entorno y con los demás.

Frente al lugar de la educación, la educación moral resalta la congruencia entre el decir y el hacer al momento de dar un juicio de valor, es así como el sujeto se hace responsable de aquello que dice y hace frente a una situación o persona; de esta forma se entiende la responsabilidad con el otro y se reconoce la capacidad del sentimiento para comunicar y establecer puentes con los otros con los cuales se comparte un espacio. Autores como Adela Cortina (1994), resaltan la capacidad del ser humano para desarrollar su vida tanto moral como política, haciendo énfasis en que el cuidado es aquel valor que permite vivir con otros, por lo que la educación en ciudadanía parte de un valor ético, tanto propio como compartido, que permite reconocer el nivel de humanidad que maneja una sociedad. Si bien la vida moral y la política se forman paralelamente en el ser humano, y procesos como el de la educación en ciudadanía propenden por dicha formación, es posible preguntarse por la educación y la práctica pedagógica. Ruiz (2000) resalta en este marco la importancia de la práctica pedagógica, ya que esta posibilita la reflexión moral, trascendiendo de una mera transmisión de saberes y hallando una relación con la vida política de los sujetos.

Teniendo en cuenta lo anterior, se podría argumentar que los sentimientos morales se relacionan con la sociedad y no atañen tan solo a lo individual, sino que reflejan el sentido de una época y lo que se espera de la 
misma; es por esto que una educación enfocada en la sensibilidad moral, más allá de exaltar el humanismo, necesario en los espacios educativos, busca pensar la época actual para transformarla, así se entendería que la formación política y en valores es más que necesaria si se piensa en el momento histórico por el que atraviesa Colombia.

\section{El lugar de la educación universitaria y la formación de estudiantes en el actual contexto colombiano}

La pregunta por la educación universitaria y la formación de estudiantes que reconozcan al otro es una pregunta actual, en tanto se relaciona con la construcción de paz y el papel de la universidad en dicha construcción. No puede hablarse de universidad sin hablar del conocimiento, en ella se produce conocimiento a través del diálogo, las ideas y las reflexiones sobre el mundo, la ciencia y el saber, dicho conocimiento permite entrever la responsabilidad social de la universidad en tanto repercute, en cierta medida, en ella; sin embargo, en la actualidad, la universidad ha perdido dicho sentido, ya que se encuentra atada a indicadores que responden exclusivamente a temas de pertinencia, y que, más allá de responder a las necesidades de los contextos socioculturales, se centra en temas meramente económicos, dejando entrever cómo la educación se convierte en un bien mercantil (Hoyos, 2009).

Si bien, la universidad surge para dar un espacio al conocimiento, en la actualidad dicho conocimiento es entendido como una inversión, como un producto que se capitaliza. De esta forma, la problematización frente al análisis de la universidad en la actualidad rescata los postulados críticos de las teorías decoloniales (De Sousa, 2004), cuestionando la importancia que da la universidad a los rankings internacionales versus la poca relevancia que da a la generación de conocimiento relevante para su contexto local y regional. La universidad entonces adquiere un carácter empresarial donde se patenta el saber y los hallazgos científicos con el fin de comercializarlos a nivel mundial.

Es por esto que es importante pensar en la identidad de las instituciones educativas partiendo de la discusión y reflexión en torno a la formación humanista (Nussbaum, 2016b). Si se parte de que la responsabilidad social de la universidad va más allá de ser un tema meramente de economía sostenible y se analiza como algo intrínseco de las instituciones, sería posible reconocer que su finalidad no se sitúa en la mera formación de profesionales útiles al mercado, sino que también se relaciona con la formación de seres humanos y ciudadanos, más si se tiene presente que todo proceso educativo debe orientarse a la formación ciudadana, esto atendiendo a la responsabilidad designada a la educación de formar al ser humano integralmente (Hoyos, 2009).
Sin embargo, ante las demandas que hace el neoliberalismo a la educación en general, queda claro que su fin no es la formación de ciudadanos, ni la sociedad, sino el mercado, de allí que se cuestione constantemente la legitimidad y relevancia de la universidad y la producción que esta hace del conocimiento. La idea de comparar a la universidad con una empresa, permite que la universidad sea entendida como un lugar donde se invierte y se espera una producción, su problema no es el estudiante, su problema es producir un conocimiento que sea rentable, esto plantea una crisis en la forma en que se entiende la universidad, y a su vez muestra un desafío enorme, pues permite ver la necesidad de replantear su finalidad en la sociedad con el fin de no desaparecer. La universidad requiere preguntarse por el entorno social, por las poblaciones, reconociendo su responsabilidad social, promoviendo el desarrollo humano y formando a sus estudiantes como ciudadanos activos que contribuyan a la sociedad (De Sousa, 2004).

Es por ello que se hace necesario retornar a las funciones centrales de la universidad que eran: cultivar el conocimiento, de allí el lugar de la investigación; formar integralmente a la persona, y tener una proyección crítica sobre la sociedad (De Sousa, 2004). Dichas funciones no pueden obedecer al mercado, pues se perdería de entrada la necesidad de formar ciudadanos críticos; al respecto, se podría decir, en palabras de Rosa Ludy $\mathrm{Arias}^{3}$, que la necesidad de formar ciudadanos críticos desde la universidad requiere de una reestructuración política y social de la misma, la cual permita visibilizar el papel de la universidad hoy, donde lo social no sea sencillamente un agregado en algunas carreras profesionales, sino un lugar que resignifique los saberes que aprenden los estudiantes en la universidad.

Si se analiza la forma en que los programas profesionales se organizan en la actualidad, es posible comprender que más allá de la responsabilidad de la universidad por formar políticamente o por producir conocimiento significativo, esta se ha venido centrando en aspectos como la cobertura y las tasas de graduados por año en sus programas, dejando de lado aspectos como la formación del ser, la producción de conocimiento y la transformación social; de allí que la educación universitaria necesite retornar al humanismo, pues desde allí podría irse formando un saber que parta del diálogo, la argumentación y la interculturalidad que confluye en Colombia (Hoyos, 2009).

Es así que la educación universitaria debe configurarse no como aquella que permite alcanzar el éxito, sino como parte fundante de la vida del ser humano y de la construcción de ciudadanos que comprendan su existencia a través de los otros, que comprendan su lugar y las

3 Comunicación personal, 17 de octubre de 2016. 
culturas que confluyen en él. Nussbaum (2012) reconoce que la ciudadanía que se propende en la educación para el nuevo humanismo debe llevar a la persona a concebirse como un ser humano vinculado a otros por lazos de reconocimiento y preocupación, para que puedan entenderse no como ciudadanos de una parte, sino como ciudadanos del mundo.

Ante este reto, cabe señalar la pertinencia por la pregunta frente a cómo desarrollar la sensibilidad moral de estudiantes universitarios, destacando el valor del arte en la educación más allá de la instrumentalización y reconociendo en este un espacio para que el sujeto establezca relaciones con la realidad de manera sensible, permitiéndole, a medida que se acerca a la obra de arte, que reconozca los vínculos que lo relacionan al otro y así pueda aportar a la construcción de paz en el país. La literatura en particular permite que dicho vínculo se dé de forma más amplia en tanto la narración ficcional permite profundizar en la emocionalidad de la persona, haciendo que el sujeto vea de otra forma aquello que valora o considera correcto (Nussbaum, 1995).

Es importante resaltar que la educación superior tiene un compromiso profundo con la construcción de ciudadanos que se desempeñen de forma creativa y constructiva en la sociedad, la universidad debe responder con ella en la construcción de un modelo social sostenible y justo para la construcción social, como lo resalta Alonso Peña (2008), es por esto que la universidad colombiana necesita replantear el papel que cumple frente a la construcción de paz en el país, ya que más allá de buscar responder a una normativa del Ministerio de Educación Nacional, debe empoderarse de un discurso que parta del conocimiento y del saber que de ella emerge para formar estudiantes que construyan, desde sus disciplinas y saberes las bases de la construcción de paz que el país requiere. No puede ser la paz un simple tema optativo dentro de la universidad, es necesario que el estudiante que haga parte de una institución universitaria, sea cual sea su profesión, reconozca la historia de Colombia, la historia del horror que ha llevado al país a hablar de paz, con el fin de que pueda, a través de su profesión, contribuir a la construcción de dicha paz.

\section{Conclusiones}

Comprender el aporte de la literatura al desarrollo de la sensibilidad moral en estudiantes universitarios, en un contexto pedagógico que contribuya a la construcción de paz, implica el reconocimiento de experiencias, estudios, procesos y proyectos que se han venido realizando sobre el tema. El recorrido por las investigaciones al respecto permite entender que el objetivo de la universidad va más allá de formar profesionales para el trabajo, puesto que resaltan la importancia de formar el carácter humano en los estudiantes que ingresan a la formación profesional; de esta forma, en la actualidad, la pregunta sobre la enseñanza universitaria en Colombia tiene una estrecha relación no solo con los saberes y disciplinas, sino con la formación ciudadana, el desarrollo de la sensibilidad moral y el contexto social en el cual están inmersos los estudiantes, con el fin de que generen espacios de convivencia que permitan la construcción y transformación del país.

La importancia de profundizar en los estudios sobre el desarrollo de la sensibilidad moral de estudiantes universitarios radica fundamentalmente en el momento sociohistórico por el cual atraviesa el país. A partir del segundo semestre del 2012, el Gobierno nacional y los representantes de las Farc ${ }^{4}$, iniciaron un trabajo en conjunto para llegar a un acuerdo que pusiera fin al conflicto armado entre las dos partes; esto llevó a pensar profundamente el tema de la paz y cómo a través de diálogos y acuerdos era posible establecer un fin a la violencia que ha aquejado al país por decenios. En este marco se reconoce la importancia del trabajo en conjunto de toda la sociedad para construir una paz sostenible que permita a todos los ciudadanos del país convivir en paz, es por esto que, respondiendo a lo anterior, y buscando generar un espacio para el aprendizaje, la reflexión y el diálogo sobre la cultura de paz, se da la expedición de la Ley 1732 de 2014, en la cual se establece la cátedra de la paz en todas las instituciones educativas del país, incluyendo la educación superior. En el caso de la universidad, la cátedra de la paz se debe desarrollar de acuerdo con su modelo educativo y sus programas académicos, teniendo presente el principio de autonomía universitaria.

Frente a este desafío, las universidades colombianas han venido desarrollando iniciativas importantes para implementar la cátedra de la paz, como lo son: foros, cursos, cátedras abiertas, conferencias y disertaciones académicas sobre el tema, en su gran mayoría impulsados por bienestar universitario, medio universitario, observatorios de derechos humanos, o unidades de proyectos estratégicos, entre otros; al igual que los movimientos estudiantiles, que generan espacios de diálogo con la comunidad universitaria y la comunidad en general, movilizaciones, folletos, muestras artísticas como performances, etc.

Todos estos esfuerzos logran generar espacios de análisis y reflexión en torno a la paz y la importancia de esta en el país, sin embargo, al ser procesos transversales y autónomos que se brindan abiertamente a la comunidad universitaria en general, difícilmente logran impactar en todos los estudiantes, ya que en ocasiones estos no llegan a conocer de dichos procesos, o estos espacios tienen cupos limitados $u$ horarios que no son factibles para todos los estudiantes, siendo ajenos al total de la población

4 Llamadas Fuerzas Armadas Revolucionarias de Colombia hasta el 31 de agosto de 2017 que se constituyen como el partido político Fuerza Alternativa Revolucionaria del Común. 
estudiantil o impactando solo a unos pocos. Un ejemplo de ello es el taller "Conciencia y relación. Habilidades sociales para la paz", ofertado para los estudiantes, desde la coordinación del programa de cultura para la paz de la Pontificia Universidad Javeriana, que en su sede de Bogotá D.C. contaba en el 2013 con 22000 estudiantes de pregrado, en sus dieciocho facultades, y cuyo cupo se reducía a tan solo 30 personas.

Por lo anterior, se hace necesario buscar estrategias que permitan brindar una formación no solo profesional, sino política a todos y cada uno de los estudiantes universitarios. No puede olvidarse que la universidad en el contexto actual, más allá de desarrollar competencias laborales, debe preocuparse por formar a sus estudiantes en y desde lo humano, con el fin de que puedan convivir, respetar y reconocer a los otros, permitiéndoles no solo comprender la importancia y trascendencia de la construcción de la paz en sus contextos, sino, a su vez, reconocer al otro y su historia como parte de sí, de lo que los constituye como seres humanos, ciudadanos y colombianos. (Hoyos, 2009).

Es por esto que es importante trabajar sobre la formación ética, emocional y en valores de los estudiantes universitarios desde lenguajes sensibles tales como los del arte, ya que estos permiten conectar tanto lo cognitivo como lo sensible del ser humano en la comprensión de las realidades, haciendo posible que los estudiantes reconozcan al otro por medio de lenguajes distintos a los meramente académicos, permitiéndoles crear lazos con el otro que se les presenta a través de las obras artísticas (Nussbaum, 2008). Entre las obras artísticas, se destaca la literatura no solo porque esta le permite al sujeto profundizar en su emocionalidad desde lo cognitivo y lo sensible, o por el valor de la literatura para la comprensión profunda del ser humano y del mundo, sino también porque en el caso puntual del estudiante universitario, este ha tenido algún acercamiento a la literatura desde los primeros años de su formación escolar o antes, dependiendo de su contexto familiar, social y cultural, lo cual facilita la mediación de la literatura en los espacios universitarios, ya que podría decirse que existe algún acercamiento por parte de los estudiantes a ella.

La necesidad de formar a los estudiantes universitarios humana y políticamente desde lugares sensibles tales como la literatura, con el fin de fortalecer no solo sus saberes disciplinares, sino su forma de relacionar dichos saberes con las realidades sociales en las cuales se encuentran inmersos, de forma que puedan transformar las complejas realidades del país, se presenta aquí como una invitación para continuar explorando en torno a la literatura, la sensibilidad moral, la educación universitaria y la construcción de paz, temas que requieren del compromiso y trabajo de investigadores y académicos que comprendan, que analicen, que desarrollen procesos, proyectos e iniciativas que sean cercanas a las realidades sociales, que estén comprometidos con la trasformación y el desarrollo social del país.

Un gran desafío para la investigación universitaria es el de pensar la universidad y el sentido de la misma dentro de la sociedad, resaltando su papel en la construcción de paz. La universidad requiere, desde la investigación y la producción de saberes, ampliar su mirada frente al tema de la paz; por ello, debe empezar a construir conocimiento para la paz en todos los niveles y escenarios de la sociedad, le corresponde formar a sus estudiantes política y humanamente, de forma que estos logren ser profesionales que asuman una responsabilidad ético-política frente al saber y frente a lo que ocurre en el país, reconociendo al otro y sus realidades. Es fundamental que la universidad potencie el trabajo académico más allá de los discursos tradicionales, fortaleciendo el reconocimiento de la historia del país, trabajando con las comunidades y dando un lugar preponderante a la sociedad, para este fin la sensibilidad moral y el desarrollo de esta a través de la literatura se convierten en espacios desde donde pueden darse los primeros pasos en este gran desafío.

\section{Referencias}

Adorno, T. (1984). Teoría estética. Madrid: Ediciones Orbis.

Alonso, J. (2008). El sentido social de la universidad. Educación Superior y Sociedad. 13(2),49-63.

Aristóteles. (trad. 1998). Ética Nicomaquea. México D.F.: Editorial Porrúa.

Congreso de la República de Colombia. (01 de septiembre de 2014). [Ley 1732 de 2014]. Recuperado de http://wsp.presidencia.gov.co/Normativa/Leyes/Documents/LEY\%201732\%20DEL\%2001\%20 DE\%20SEPTIEMBRE\%20DE\%202014.pdf. DOI: https://doi.org/10.5962/bhl.title.65017

Congreso de la República de Colombia. (28 de diciembre de 1992). [Ley 30 de 1992]. Recuperado de https:// www.cna.gov.co/1741/articles-186370_ley_3092. pdf. DOI: https://doi.org/10.2307/j.ctvk8vzh8.3

Cortina, A. (1994). Ética de la empresa: claves para una nueva cultura empresarial. Madrid: Editorial Trotta.

De Sousa, B. (2004). La universidad del siglo XXI. Para una reforma democrática y emancipadora de la universidad. La Paz: Plural Editores. DOI: https://doi. org/10.3989/redc.2013.4.1046

Hoyos, G. (2009). Educación para un nuevo humanismo. Magis, 2, pp. 425-433.

Nussbaum, M. (1995). Justicia poética. La imaginación literaria y la vida pública. Barcelona: Editorial Andrés Bello. DOI: https://doi.org/10.3989/isegoria.1995. i11.254

Nussbaum, M. (2008). Paisajes del pensamiento. La inteligencia de las emociones. Barcelona: Editorial Paidós. 
Nussbaum, M. (2010). Sin fines de lucro. Por qué la democracia necesita de las humanidades. Buenos Aires: Katz Editores. DOI: https://doi.org/10.15304/ ag.32.2.1432

Nussbaum, M. (2012). Crear capacidades. Propuesta para eldesarrollohumano. Barcelona:EditorialPaidós.DOI: https://doi.org/10.5354/0717-8883.2014.31834

Nussbaum, M. (2014). Las emociones politicas. ¿Por qué el amor es importante para la justicia? Barcelona: Editorial Paidós. DOI: https://doi.org/10.18601/16578651. n16.11

Nussbaum, M. (2016a). Inclusión, equidad y mercado: ¿objetivos de la educación superior? Nómadas, 44,13- 25.
Nussbaum, M. (2016b). Una carta para el pueblo colombiano. En: P. Gentili, S. Alvarado y E. Rueda Barrera. (Eds.), Paz en Colombia. Perspectivas, desafios, opciones. (pp. 17-19). Buenos Aires: Clacso.

Ruiz, A. y Quintero, M. (2000). Análisis desde la ética de la responsabilidad, de los criterios valorativos, éticos, politicos y pedagógicos en los procesos de formación en valores. Bogotá: Funvhec y Colciencias. DOI: https://doi. org/10.2307/j.ctvb6v5hs.10

Sábato, E. (1976). El escritory sus fantasmas. Buenos aires: Emecé Editores.

Sábato, E. (2000). La resistencia. Buenos Aires: Grupo Planeta. 